\title{
Phénomènes hydrauliques liés au mouvement des péniches dans les voies navigables
}

\author{
Application particulière à la stabilité des berges des canaux
}

\section{Hydraulic phenomena associated with the movement of barges in navigable waterways}

\author{
PAR P. LHERMITTEE
}

INGÉNEUR DES PONTS ET GHAUSSËES

DIRECTEUR TECHNQUE DU LABORATOIRE CENTAA D'HYORAULYOUE DE FIANGE

\begin{abstract}
Cetle communication expose les conclusions théoriques qu'il est possible de tirer de recherches sur modèle réduit, effectuées an Laboratoire Central d'Hydraulique de France, concernant le problème de l'érosion des berges des canaux de navigation intérieure.

Les résnltats qui sont exposés ei-après, concer. nant les phénomènes hydrauliques et les moulvements de matériaux lies aux monvements des péniches et des automoteurs, ne prétendent pas épuiser ce problème, jusqu'alors relativement peu étndié. Ils contiennent, en particulier, des renseignements originaux, mettant on évidence l'intérêt des essais sur modèle réduit dans l'étude de problèmes de ce genre. En outre, il est proposé, à titre indicatif, certains essais qu'il serait interessant d'entreprendre en continuation de ees recherches.

La conclusion de ces études permet d'énoncer des applications pratiques, concernant la protection, et les formes, des cuvettes des canaux de navigation.
\end{abstract}

\begin{abstract}
In his paper, the Author gives an expose of the theoretical conclusions that can be drawn from scale model research carried ont at the Laboratoire Central d'Hydraulique de France on the problem of bank erosion in inland waterways.

The results given in the article in connection with hydraulic phenomena and the movement of solid materials related to the movements of towed or, self-propelled barges in no way claim to represent an exhaustive solution for a hitherto relatively little studied problem; however, they do contain original information that brings the value of scale research for problems of this type well into evidence. Furthermore, some interesting suggestions are made as to tests that would seem well worth while carrying out for future research on the subject.

In conclusion, the Author gives an insight into some practical applications of this work, in connection with the protection and form design of navigable channel sections.
\end{abstract}

L'évolution des péniches tractées et des péniches automotrices dans les rivières navigables ou dans les canaux de navigation, engendre des phénomènes hydrauliques relativement complexes, qui dépendent de la forme et du tonnage des péniches, de la section transversale des rivières ou des canaux (surface et configuration), de la vitesse de transit, de la puissance des hélices, etc. Ceux-ci réagissent sur la vitesse de la péniche et sur la puissance absorbée, ainsi que sur la stabilité des berges et du plafond de la rivière ou du canal.
Ce problème faisait l'objet de la communication $n^{\circ} 1$ de Navigation intérieure du XVIII ${ }^{\circ}$ Congrès International de Navigation, à Rome, en 1953. L'ensemble des communications présentées à ce Congrès et celles présentées au Congrès de Lisbonne en 1949, ont apporté sur cette question des précisions et des données particulièrement intéressantes. Le présent exposé a seulement pour objet de développer quelques points particuliers de ce vaste programme en complément direct des théories d'ensemble exposées dans ces communications. 


\section{A) EXPOSÉ DES ESSAIS}

En effet, le Laboratoire Central d'Hydraulique de France a eu l'occasion d'étudier, à la demande des Dragages de la Haute-Moselle et en liaison avec le Service de la Navigation des Ponts et Chaussées de Nancy, l'action d'érosion conséculive au passage de péniches tractées ou de péniches automotrices sur les berges des canaux de navigation.

L'étude effectuée au L.C.H.F. (fig. 1 et 2), comportant la représentation de canaux de navi- demande la réalisation d'essais systématiques avec des matériaux de densité inférieure à celle des matériaux nature; et ce en particulier pour pouvoir utiliser des maquettes à plus petite échelle.

Au cours de ces essais, nous avons observé et analysé les divers phénomènes hydrauliques et d'érosion dans les différents cas suivants :

- Passage de péniches tractées à vitesse relativement élevée;

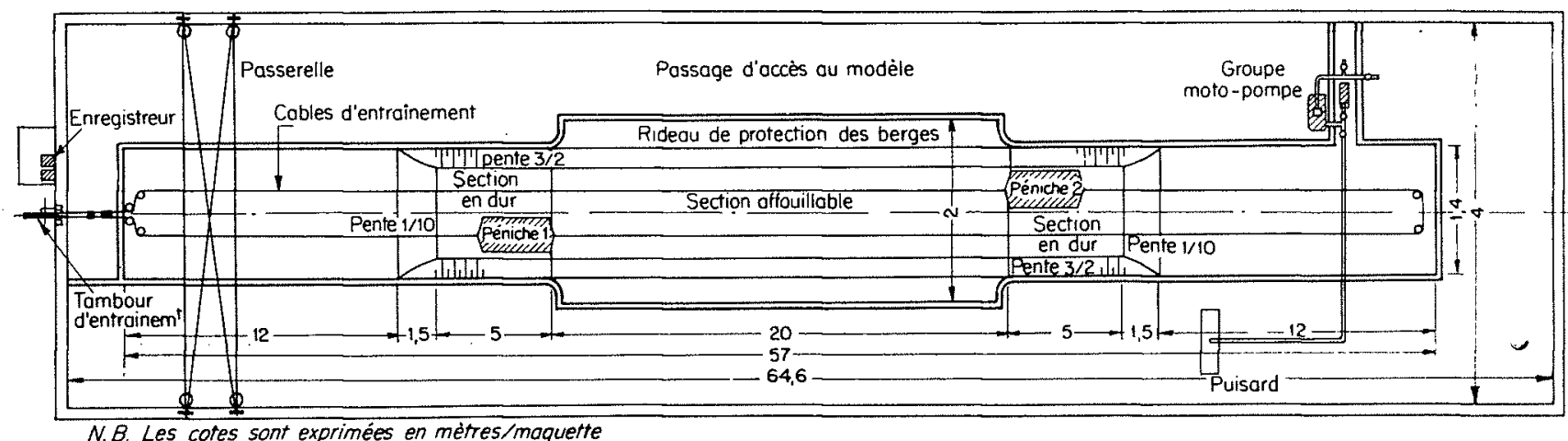

Fig. 1.

Plan de la maquette. (Nota : le plan cidessus comporte une distorsion de 4 entre les dimensions longitudinale: et les dimensions transversales.)

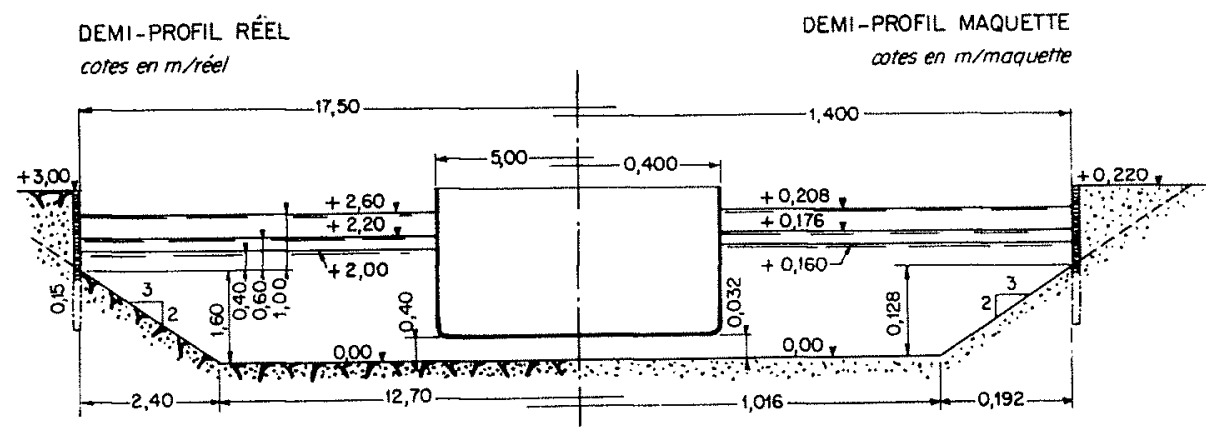

FIa. 2

Profil transversal du canal.

gation intérieure, de dimensions modestes, a permis d'adopter une échelle de $1: 12,5$; ceci présente un énorme avantage en ce qui concerne l'étude de l'érosion, car la représentation fidèle en modèle des mouvements du matériau constitutif des berges est, de ce fait, grandement facilitée. En effet, nous avons pu, avec des sables naturels de granulométrie adéquate, représenter convenablement les formes d'équilibre des berges lors de passages des bateaux. Précisons, tontefois, qu'une telle étude ne représente, à nos yeux, qu'une première phase de l'étude d'ensemble de l'érosion des berges des canaux sous l'effet du batillage et que, l'exploitation rationnelle des modèles réduits dans l'étude de cette question
- Croisement de péniches traclées;

- Cas particulier des automoteurs;

-... Influence du tirant d'eau.

Les études que nous avons effectuées ont été réalisées à l'aide de modòles qui représentaient, à l'échelle de $1: 12,5$, les péniches flamandes lype, circulant sur les canaux de navigation de l'est et du nord de la France.

Dans le cas de péniches tractées, nous avons admis volontairement des formes extrêmement massives correspondant à un bloc coefficient do l'ordre de 0,98 , bien que de telles péniches ne soient plus utilisées sur nos canaux.

De même, pour les essais en auto-moteurs, alors que le bloc coefficient de ces bâtiments 
varie normalement entre 0,89 et 0,93 , la péniche utilisée représentait le modèle réduit à l'échelle de $1: 12,5$ des péniches transformables, actuellement construites par la Société de Reconstruction et de Renouvellement du Parc Fluvial, et correspondant à un bloc coefficient de 0,94.

Précisons, enfin, qué nos essais avaient sur- tout pour but l'étude de l'érosion des berges des canaux et la vérification de l'efficacité de certains dispositifs de revètement.

Nous exposerons tout d'abord les observations particulières qui ont été faites en ce qui concerne les phénomènes hydrauliques eux-mêmes, puis celles relatives aux phénomènes d'érosion.

\section{B) PHÉNOMÈNES HYDRAULIQUES}

\section{1. - Péniches tractées.}

L'évolution d'une péniche tractée s'accompagne des phénomènes hydrauliques classiques bien connus, caractéristiques de toute évolution de navire dans un canal :

- Génération de deux systèmes d'ondes (quo nous désignerons sous le vocable d'ondes de batillage), le plus important étant celui qui prend naissance à l'avant de la péniche, le second étant engendré après le passage de la péniche;

- Modification de la surface libre du canal: formation d'un bourrelet à l'avant de la péniche et abaissement du plan d'eau au droit de la péniche correspondant à la mise en vitesse des courants de retour (fig. 3);

- Corrélativement à l'abaissement du plan d'eau, enfoncement de la péniche; de tourbillons alternés qui se détachent alternativement de part et d'autre de la péniche. Derrière la péniche se déplace avec celle-ci une zone de contre-courants et de turbulence intense provenant de la discontinuité des formes du bâtiment et correspondant, en fait, à une augmentation purement hydraulique de la carène de celuici, avec entraînement de masses d'eau non négligeables.

Sur le fond et les murailles de la péniche, se développe une couche-limite relativement mal connue, mais qui peut avoir une importance considérable sur la résistance à l'avancement, eı égard à la faible profondeur d'eau restant entre la péniche et le plafond du canal, par suite de l'enfoncement de cette dernière; en effet, pour des vitesses insuffisantes, cette couche-limite rejoint la couche-limite propre au canal s'opposant à l'établissement des courants de retour.

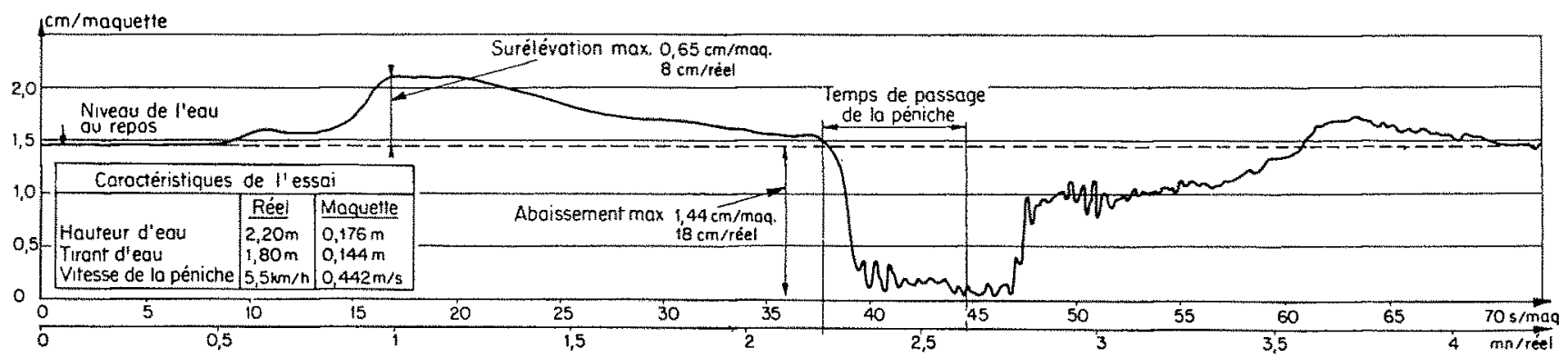

Fic. 3.

Variation de la ligne d'ean au passage, dans l'axe du canal, d'une péniche tratée.

- Génération de courants de retour dirigés dans le sens inverse de la péniche afin d'assurer l'échange des volumes d'eau, entre l'avant et l'arrière de la péniche, correspondant au déplacement de la péniche.

Ces éléments peuvent être mis en évidence par le calcul, ainsi que l'a développé en particulier M. Shijf.

De plus, dans le cas particulier des péniches tractées de nos canaux, à forme parallélépipédique, on enregistre la formation d'une double file

\section{2. - Croisement de deux péniches.}

Lors du croisement de deux péniches, les phénomènes hydrauliques présentent un caractère sensiblement différent. On enregistre tout d'abord un gonflement et un étalement de l'onde existant devant les péniches (fig. 4) lorsque celles-ci se rapprochent, puis un abaissement et une stabilisation relative du plan d'eau qui se maintient à peu près constante lorsque les péniches sont à la même hauteur. Lorsque les deux péniches se séparent, on enregistre une brutale dénivellation 


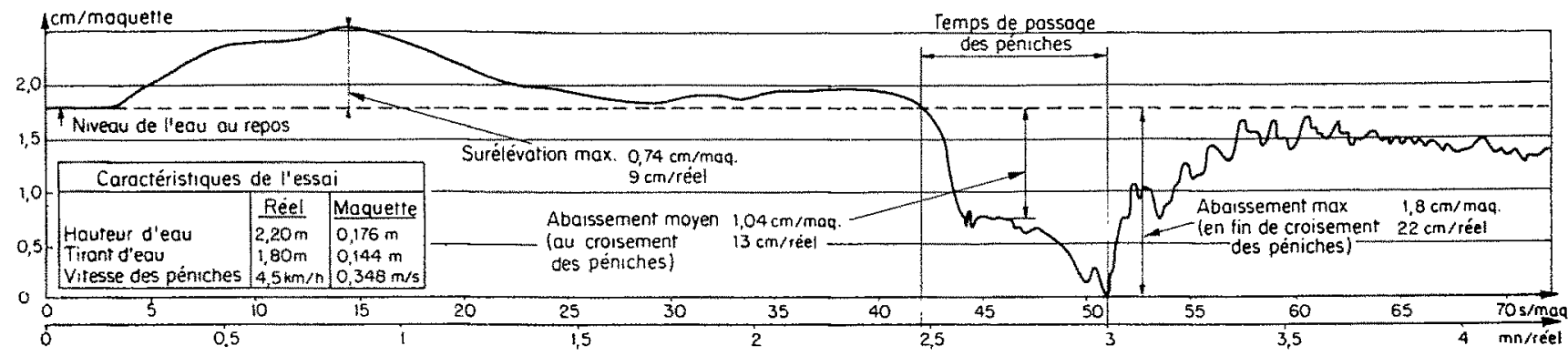

Fig. 4.

Variations de la ligne d'eau au croisement de deux péniches tractés.

du plan d'eau el un rétablissement progressif a la hauteur normale avec des oscillations moins importantes que pour une seule péniche.

Dans ce cas (et en particulier les péniches se croisant à la même vitesse, ce qui correspond aux conditions d'expérience), les phénomènes secondaires exposés précédemment se trouvent profondement modifiés car, lorsque les deux péniches se rencontrent, il n'y a plus d'échange d'eau entre l'avant d'une péniche et l'arrière de l'autre.

Par contre, lorsque les deux péniches se sćparent, il y a, à ce momenl, un brutal appel d'eau dans la zone centrale et c'est ce qui engendre la brusque oscillation que l'on enregistre.

L'abaissement du plan d'eau est donc proporl'omellement moindre, mais il se produit, par contre, un mouvement d'ean circulaire qui ausimente considérablement les effets secondaires. Dans l'ensemble, le croisement des deux péniches est, malgre tout, beaucoup moins néfaste que le passage d'une péniche seule en ce quí concerne les phénomènes liés aux conditions d'écoulement hydraulique (réaction sur la péniche, érosion des herges, absorption des puissances) malgré la faible distance séparant la muraille de la péniche de la berge.

Par contre, on comprend que le trématage des pénicines, qui revient à doubler l'effet de piston provoqué par une péniche, ait tendance à augmenter considérablement l'abaissement du plan d'eau et l'influence des courants de retour. II s'ensuit comme corollaire que le phénomène d'aspiration constaté entre deux péniches qui trématent est absolument normal.

\section{3. - Phénomènes d'écoulement hydraulique propre aux automoteurs.}

Si, dans l'ensemble, les phénomènes d'ćcoulement hydraulique des automoteurs sont semblables à ceux des péniches, ils sont, malgré tout, profondément modifićs par la présence de lhé lice. L'hélice agit non seulement par la création de remous et de turbulence à l'arrière du navire, mais également par un appel d'eau depuis l'avant du navire, afin d'assurer l'alimentation de l'hélice. Cet appel d'eau favorise, en fait, l'échange d'eau nécessaire entre l'avant et l'arrière de la péniche; il en résulte une modification de l'assiette même du navire, dont, le plus souvent, l'arrière enfonce plus que l'avant, et une modification sensible des courants de relour.

Si ceux-ci sont comparables à l'avant même du navire, ils ont tendance à converger vers l'hélice, d'où une augmentation importante de leur's composantes verticales, et surtout une suppression quasi totale à partir du niveau de thélice.

On concoit que les conditions de bonne navigation seront donc profondément differentes el que les actions d'érosion pourront être complètemenl modifiées.

En particulier, la thérie classique, qui permel de démontrer que, dans le cas d'ume péniche tractée, les meilleures conditions de navigation correspondent au plus grand rayon hydraulique du canal, c'est-à-dire à une forme rectangulaire, n'est plus lotalement vraie pour la péniche automotrice.

En effet, le phénomène de propagation gravitaire d'ondes n'est plus alors seul à déterminer le rendement de l'effort propulsif, et l'aisance aux bouchains et sur le fond, lice à l'alimentation convenable de l'hélice, est, dans ce cas, encore plus importante.

Ces remarques s'appliquent essentiellement aux automoteurs à pleine charge dont l'hélice agit en profondeur et pour lesquels le rapport des sections mouillées du canal et de la péniche est faible. Pour l'automoteur lège ou a mi-charge. dont lhélice se situe près de la surface et dont la vitesse de croisière n'est plus limitée par la vitesse limite, l'importance du remous en surface est très augmentée et le courant de retour peut être lui-même relativement plus important (cas particulier des automoteurs lèges ou à mi-charge qui trématent une autre péniche), ce qui peut provoquer des érosions intenses des berges. 


\section{C) EROSION DES BERGES}

L'observation des phénomènes hydrauliques qui accompagnent le mouvement des péniches, et leur analyse, permet d'expliquer les phénomènes d'érosion consécutifs au déplacement des péniches que l'on constate, en nature, sur les berges des canaux ou des rivières, et que nous avons reproduits dans des essais qui ont été effectués au L.C.H.F.

Pour bien comprendre l'ensemble de ces phénomènes, il importe de préciser que les mouvements de matériaux dans les rivières dépendent, non seulement de la vitesse du courant, mais essentiellement de la turbulence interne du courant.

En fait, la notion de vitesse d'entraînement telle qu'elle est habituellement définie n'a pas de sens très précis. Dans le cas d'écoulement réellement laminaire, les vitesses nécessaires pour obtenir l'entraìnement du matériau sont, en fait, extrêmement importantes, et lorsque l'on détermine, dans le langage usuel, les vitesses critiques d'entraînement des matériaux, on fait en général allusion à la vitesse nécessaire pour créer autour du matériau une zone de turbulence suffisante pour que les fluctuations de pression permettent la mise en saltation des éléments.

En ce qui concerne les phénomènes hydrauliques liés au mouvement des péniches, si l'on fait abstraction de la turbulence, les seuils d'érosion seraient définis par les caractéristiques du seul courant qui existe dans le canal : le courant de retour. Or, on constate que les vitesses mesurées du courant de retour dans un canal sont inférieures à ce que l'on a l'habitude d'appeler les vitesses critiques d'entraînement des matériaux constituant les berges des canaux. D'autre part, le courant de retour est, en général, plus violent avec les profils habituels des canaux au droit des bouchains ou au fond, qu'en surface. Or, les érosions que l'on constate en nature sont particulièrement importantes à la surface de l'eau, alors qu'elles sont relativement faibles au fond.

Il apparaît donc que le critère essentiel, en ce qui concerne la mise en mouvements du matériau constituant les berges des rivières ou des canaux de navigation, est la turbulence et non pas le courant de retour. Le courant de retour, ou les courants oscillatoires perpendiculaires à la direction du navire, n'ont pour action que de transporter les matériaux précédemment mis en saltation dans les zones de turbulence importante.

Or, il existe quatre zones de turbulence caractérisées :

- Zone d'influence des ondes de batillage qui est limitée à la surface du canal : l'énergie de ces ondes se détruisant principalement par déferlement ou par modification de la structure interne près des berges, dans les zones où les profondeurs d'eau sont faibles;

- Sillage de l'hélice;

- Zone des lignes de tourbillons alternés, située à l'arrière de la péniche;

- Conche turbulente développée sur le fond de la péniche et sur le plafond du canal.

En fait, les zones de turbulence particulièrement actives sont essentiellement les zones 1 et 2 ; suivant qu'il existe dans la zone considérée des courants importants ou non, on observera le déplacement des matériaux ou la formation de rides. Les zones 3 et 4 n'ont qu'une action partielle sur les éléments fins qui sont mis en suspension, mais ne sont pas sujets à des déplacements importants.

Les essais de laboratoire relatifs aux érosions des berges des canaux de navigation ont été effectués avec deux sables de granulométrie différente :

- L'un, de courbe granulométrique relativement resserrée, avait un diamètre moyen de $0,50 \mathrm{~mm}$;

- L'autre, présentant une courbe granulomémétrique plus dispersée, avait un diamètre moyen de $0,62 \mathrm{~mm}$.

La correspondance entre ces matériaux-maquettes et le matériau-nature est délicate à préciser. On sait, en effet, que pour cet ordre de grandeur des diamètres, la similitude de Froude n'est plus directement applicable, et l'on doit tenir compte d'un coefficient correcteur.

Les deux matériaux correspondaient pratiquement à deux matériaux en nature, l'un relativement fin $\left(D_{50}: 5 \mathrm{~mm}\right)$ et peu dispersé $(0,8 \dot{a}$ $18 \mathrm{~mm}$ ), l'autre correspondant à un remblai graveleux tel qu'on en rencontre fréquemment le long des berges des canaux de navigation de la France $\left(\mathrm{D}_{\overline{7} 0}: 6,2 \mathrm{~mm} ; 0,5<\mathrm{D}<65 \mathrm{~mm}\right)$.

\section{1. - Phénomènes d'érosion provoqués par les passages des péniches tractées.}

Les péniches tractées provoquent une érosion importante dans la partie supérieure des berges des canaux. Les ondes de batillage ont, en effet, une action puissante dans cette zone et assurent la mise en suspension des éléments de dimensions faibles ou moyennes.

L'existence d'un courant de retour important entraîne ces éléments relativement loin, et ceuxci se déposent, de façon à peu près uniforme, sur les berges et le fond du canal.

Le départ des éléments fins a pour résultal de déchausser les éléments plus grossiers qui roulent en général au pied du talus. Les dimen- 


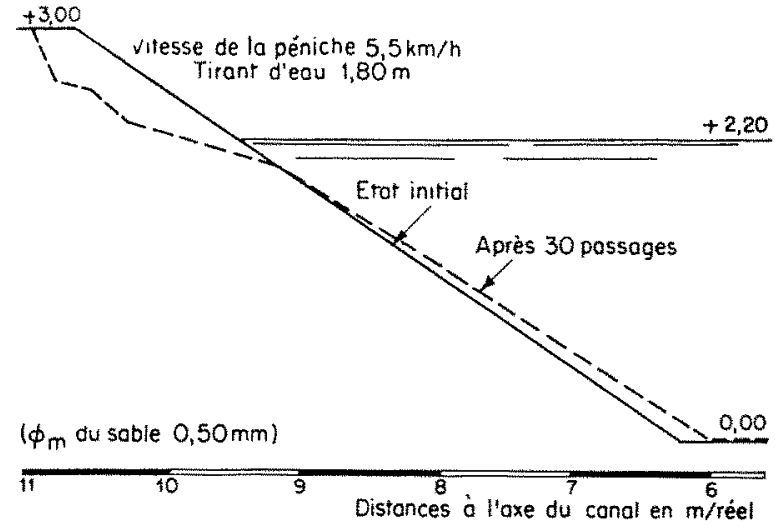

Fic. 5 .

Erosion des herges au passage d'une péniche dans laxe.

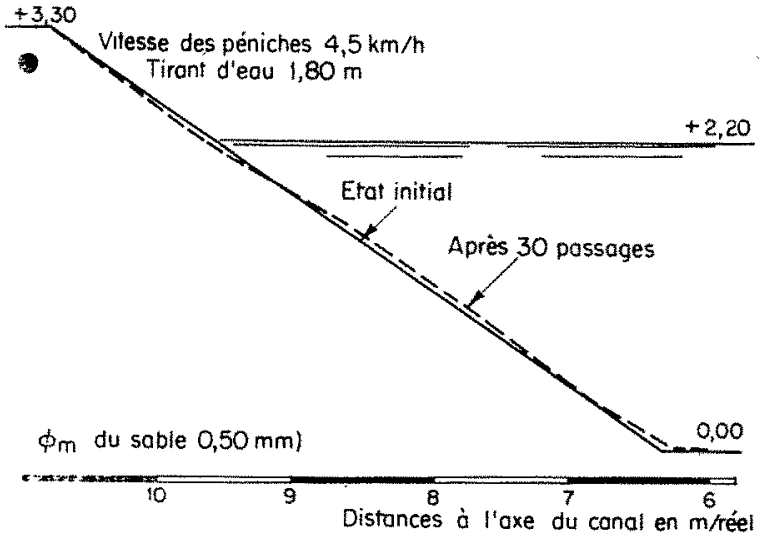

Fic. 6 .

Erosion des herges au croisement des péniches tractées.

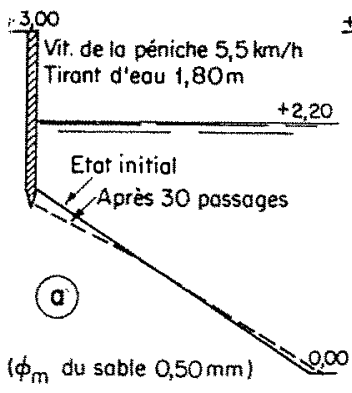
Dist ò l'axe du canal en m/réel Frosion des berges an passage d'une péniche dans laxe.

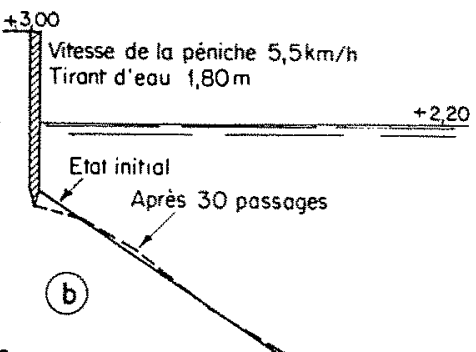

$\phi$

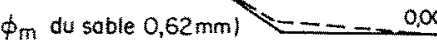

Fir. 7
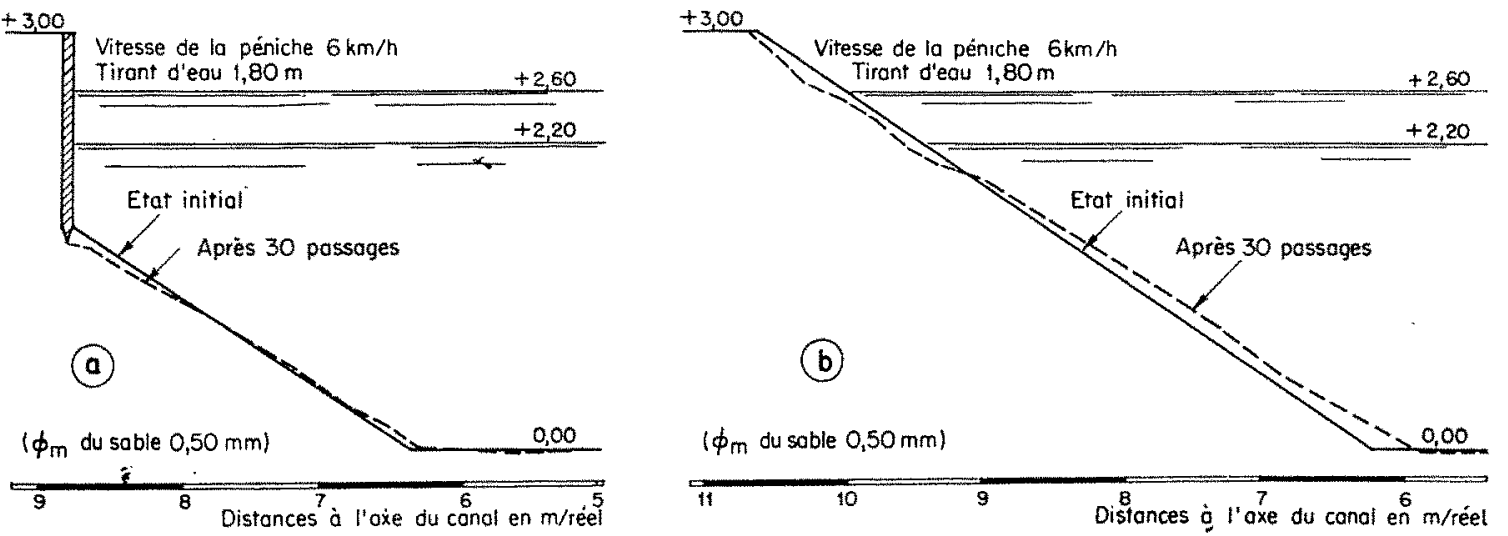

Fic. 9.

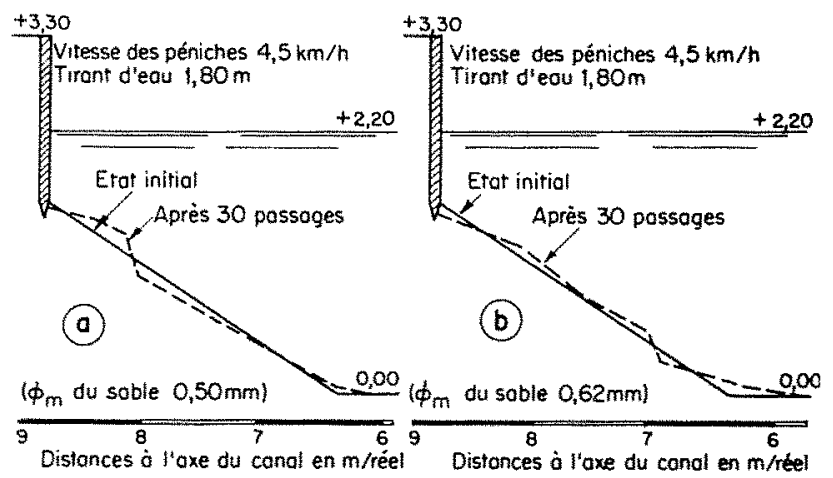

Fic. 8.

Erosion des berges au croisement des péniches fractés.

Erosion des berges au passage d'une péniche automotrice.

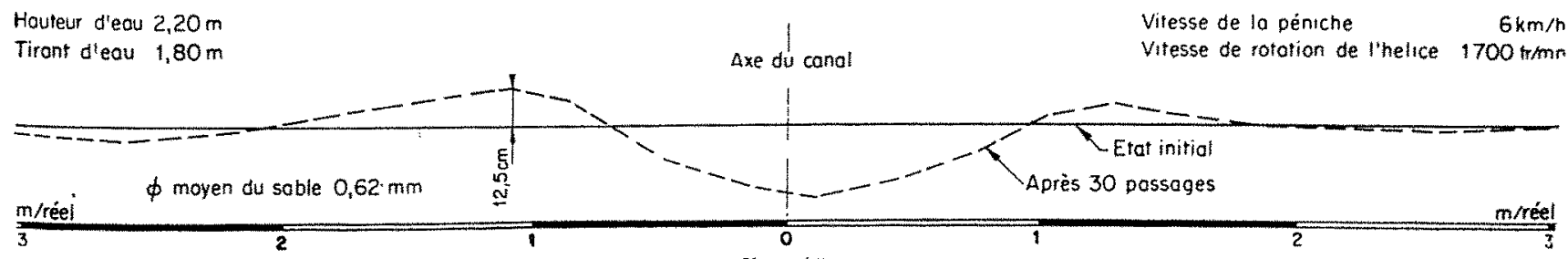

Altération du fond du canal engendrée par le remous de hélices. 
sions des éléments entraînés sont évidemment fonction de la nature du transit.

L'action, sur le fond, des péniches tractées est relativement plus faible. Seuls les éléments fins sont mis en suspension dans la zone centrale, par suite de la présence de la couche turbulente de fond, mais, au droit des denx files de tourbillons alternés, il peut se produire des mises en suspension plus importantes.

Dans les conditions de nos essais (vitesse de $5,5 \mathrm{~km} / \mathrm{h}$ et $6 \mathrm{~km} / \mathrm{h}$ ), la péniche passant rigoureusement toujours au même endroit, il s'est produit un creusement caractéristique dans les zones correspondant à cette file de tourbillons. En nature, ce phénomène intéresse, en fait, une large partie du centre du canal, ce qui, lié à l'action érosive des hélices des automoteurs (que nous étudierons plus loin), entraîne le creuse. ment, suivant une forme parabolique, du fond du canal, phénomène qui a été très souvent observé en nature.

Il est important de noter que la seule action dangereuse se limite à une zone bien délimitée qui est celle dans laquelle les ondes de batillage sont capables de mettre les éléments fins en suspension. Cette remarque confirme le fait qu'un revêtement superficiel exécuté de facon par. tielle sur les berges des canaux est souvent sufsant pour supprimer les érosions importantes.

Notons également que, les ondes de batillage étant capables de se propager très loin, il est normal qu'elles proroquent l'érosion des berges des rivières, même si l'on peut considérer que, dans celles-ci, l'évolution des péniches puisse être considérée pratiquement comme en eau libre, et, par conséquent, sans que l'existence des courants de retour ait une influence sur les berges, le courant propre de la rivière assurant le déplacement des matériaux mis en saltation.

\section{2. - Cas particulier du croisement de deux péniches.}

Le croisement de deux péniches donne lieu à un réseau d'ondes de batillage infiniment plus complexe que lors d'une seule péniche, mais, par contre, ainsi que nous l'avons yu précédemment, les courants de retour sont relativement moins importants. II existe, par contre, des mouvements secondaires qui changent totalement l'allure des mouvements de matériaux.

Dans les conditions de réalisation de nos expériences, nos péniches se croisaient toujours au même point et sans qu'entre deux croisements on effectue, comme en nature, un nombre suffisant de passages de péniches isolées. Nous étions donc dans des conditions très éloignées des conditions naturelles, mais le résultat des expériences est extrêmement instructif. Signalons également que ces croisements étaient effec- tués à allure réduite : $4,5 \mathrm{~km} / \mathrm{h}$, ainsi que cela se fait en nature.

L'évolution, au cours de ces essais, des berges de canal non situées au point de croisement est conforme aux expériences précédentes, mais on observe par contre, au droit du point de croisement, la formation de rides très nettement accusées ef une évolution beaucoup plus rapide vers un profil parabolique.

Ces phénomènes s'expliquent par le fait que l'importante énergie dispersée par turbulence dans les zones d'eau peu profonde assure la mise en saltation des éléments fins, mais l'absence de courants de retour laisse ces éléments dans la zone même où ils sont mis en suspension. Les passages des péniches se faisant toujours à la même vitesse, on concoit qu'un régime stationnaire de rides s'établisse au lieu où régulièrement le matériau est mis en suspension.

\section{3. - Péniches automotrices.}

Le passage des péniches antomotrices à pleine charge à une vitesse égale à celle des péniches tractées, produit relativement moins d'érosion sur les berges des canaux qu'une péniche tractée.

Ce résultat, qui nous avait été affirmé par certains ingénieurs des Services de la Navigation, semble a priori surprenant.

La turbulence propre à l'hélice n'apporte, en effet, que peu d'énergie facilitant la mise en saltation des éléments des berges, et, de toute façon, le sillage de l'hélice ne peut atteindre les berges qu'en des zones où le courant de retour est très atténué, et, par conséquent, où le transport des éléments est impossible.

De plus, ainsi que nous l'avons vu dans l'étude hydraulique, la présence de l'hélice a pour effet, étant donné son rôle d'aspiration des filets liquides, de diminuer l'importance des courants de surface. Ceux-ci ne sont sensibles en surface, près des berges, que sur une longueur correspondant environ aux deux tiers de la longueur de la péniche. Or, dans cette zone, les ondes de batillage n'ont pas encore atteint la berge et la mise en suspension des éléments n'a pas encore été réalisce. Au niveau de l'hélice, les courants de retour sont presque totalement supprimés. Il est donc normal que l'on observe, à vitesse égale, une évolution beaucoup plus lente, et un profil d'érosion moins accusé pour les péniches automotrices que les péniches tractées.

Par contre, le sillage de l'hélice crée une zone turbulente très importante au voisinage du fond du canal et assure la mise en suspension des éléments fins qui sont transportés par le courant d'eau créé par l'hélice. On observe alors l'ćrosion du lit central et le rejet des éléments sur les côtés.

D'autre part, le passage de péniches auto- 
motrices, à demi-charge ou lèges, est particulièrement néfaste si les limitations de vitesses imposées ne sont pas respectées. Dans ce cas, le remous de l'hélice se propage en surface, et les ondes d'accompagnement ont une action particulièrement importante et néfaste.

\section{D) CONCLUSIONS CONCERNANT LA PROTECTION ET LES FORMES DES CUVETTES DES CANAUX DE NAVIGATION}

De ces explications théoriques des phénomènes accompagnant l'évolution des péniches dans les rivières et plus particulièrement dans les canaux de navigation, nous voudrions tirer quelques applications pratiques.

1. - - Tout d'abord, la protection des berges des canaux peut se limiter à la profondeur à partir de laquelle les ondes de batillage maximum, qui peuvent être engendrées dans le canal, étant donné les conditions de navigation, sont susceptibles de mettre en suspension une partie non négligeable des éléments en place.

Cette limite peut se déterminer d'une façon précise :

- par des études théoriques et systématiques;

- par l'observation des résultats obtenus en nature

- et par des expériences en modèle réduit.

Une solution exacte de ce problème permettrait de limiter à leur strict minimum les cồteuses dépenses de revêtement des berges.

2. - L'analyse des phénomènes tend, sauf cas particulier, à condamner l'utilisation des banquettes de protection, car l'existence de ces banquettes ne supprime pas la nécessité d'un revêtement de berges; d'autre part, l'exécution de ces banquettes exige de pouvoir donner une assiette suffisante au canal de navigation, ce qui est rarement le cas pour les voies navigables de la France.

3. - Un approfondissement central de la section du canal est très souvent rentable :
- par l'augmentation de section qu'il procure;

- par l'obtention d'une meilleure alimentation en eau des hélices:

- par la diminution de résistance à l'avancement due aux couches turbulentes, etc.

Dans le cas souvent réalisé en nature où la largeur en surface d'un canal est limitée par les conditions naturelles (parois rocheuses, voies ferrées, routes), ainsi que cela se rencontre fréquemment en France, l'amélioration la plus économique du canal consistera donc à effectuer un revêtement de parois jusqu'à la profondeur correspondant aux conditions ci-dessus définies, et à draguer le canal en donnant jusqu'au plafond de celui-ci une pente conservant la stabilité des éléments en place et assurant une surprofondeur dans la partie centrale du plafond, surprofondeur réalisée tout en restant à l'extérieur de la pente naturelle du terrain.

Les remarques précédentes ne prétendent pas épuiser le sujet. De nombreuses observations en nature, ainsi que des essais complémentaires en laboratoire, seraient extrêmement intéressants pour donner plus de valeurs numériques relatives aux phénomènes décrits; mais il semble que, dans chaque cas particulier, la recherche de la solution la plus économique d'amélioration d'un canal devrait permettre de déterminer le type de protection des berges adéquat, la distance à laquelle il doit être réalisé à partir de l'axe de la cuvette, et l'influence quantitative de la vitesselimite autorisée aux péniches, sur les dépenses de protection ou d'entretien.

\section{I S C U S I O N}

Président: M. de Rouvilue

II. le Président indique que cette communication a déjà été présentée à la Section d'Hydraulique Fluviale et Maritime; il félicite M. Lhermitre pour la qualité de son exposé où l'on trouve des observations qui ouvrent la porte, soit à d'autres essais, soit à des réflexions.

M. Georges WeIL, Président, Directeur Général de la Compagnie Générale de Traction sur les Voies-Navigables, Membre de la Section d'Hydraulique Fluviale et Maritime, donne lecture de la lettre ci-après, qu'il a adressée à M. de Rouvilue, Président de cette Section, après la réunion du 28 Février 1957, à laquelle M. LhenMITTE avait déjà présenté cette communication.

Lettre de M. Georges WEILL, en date du 4 juin 1957. Mon cher Président,

Vous avez très judicieusement et immédiatement fait constater (12. de vos remarques) que les resultats de 
Ia comparaison entre péniches tractées et automoteurs semblaient contraires à l'opinion de certains spécialistes. C'est pour éviter des jugements s'écartant de l'expérience de spécialistes, que je crois utile d'intervenir pour ne pas laisser s'aceréditer des opinions, apparemment sans bases réelles suffisantes.

\section{Du point de vue des principes}

1. Les essais ont été entrepris, selon l'auteur du mémoire, dans le but d'étudier l'efficacité de certains dispositifs de revêtement pour lutter contre l'érosion des berges. Il en a été tiré des conclusions sur la comparaison des trosions dues anx bateanx automoteurs ef tractés. L'auteur sera certainement le premier à reconnaître que ses conclusions ne peuvent pas avoir la valeur de celles qui résulteraient d'essais ayant pour but essentiel la comparaison des modes de propulsion, car de tels essais auraient certainement été poursuivis de facon différente.

$2^{\circ}$ C'est ainsi que le choix des formes de bateaux soumises aux essais est différent dans l'un ot l'autre cas (coefficient 0,98 pour les tractés et 0,945 pour les automotcurs).

$3^{\circ}$ Et l'on indique par aillemrs que les péniches à coefficient 0,98 ne sont plus utilisées sur nos camaux!

$4^{\circ}$ Enfin, il semble que, autant il est insisté sur l'avintage du creusement des canaux par hélice de lautomoteur, chargé, autant les dégâts causés par l'automoteur lège, s'ils sont signalés, le sont avec une certaine discrétion.

\section{Du point de vue des faits}

1" Si les essais ont été réalisés en parois yerticales, (comme vous l'avez bien constaté ( $1^{\circ}$ de vos remarques), et si l'auteur du rapport reconnait que ce procédé est suffisant en ce qui concerne la réflexion des ondes, il est précisé en même temps que le bnt de l'étude ctrait de vérifier l'efficacité du revêtement. Pourtant, il en déduit d'autres conséquences. Or en fait, les canaux sont encore, de façon générale, ctablis avee des berges en pente.

$2^{\circ}$ Les essais correspondent à wne profondeur d'eau de $2,60 \mathrm{~m}$ at a $\mathrm{un}$ enfoncement du bateau de $2,20 \mathrm{~m}$. En fait, combien de voies navigables profitent-elles actuellement d'une telle profondeur d'eau permettant la navigation a $2,20 \mathrm{~m}$ ?

$3^{\circ}$ Les croisements ont toujours été provoqués au même endroit; or, en fait, les croisements se produisent n'importe où.

$4^{\circ}$ Il est précisé que le creusement du canal est au moins aussi intéressant que l'clargissement. On peut laccepter pour le Canal de Suez ou pour un canal généreusement conditionné. Ce n'est peut-être pas le cas de notrc réseau vétuste. En fait, en 1925 , je crois me rappeler qu'un service de remorquage fut installe sur le Canal de Bourbourg près de Dunkerque. Très rapidement, ]a seetion prit un profll presque triangulaire; les matériaux mis en suspension se déposaient le long des berges et le eroisement régulier des bateaux ne ponvait plus se faire. Il fallut d'urgence changer la méthode d'exploitation et remplacer les remorqueurs par des tracteurs.

$5^{\circ}$ Nulle part n'est indiquée la vitesse de circulation adoptée au cours des essais. On ne mentionne la vitesse de $4 \mathrm{Km} / \mathrm{h}$ qu'au moment des croisements et elle est alors considérée comme «allure réduite». On peut donc supposer que la vitesse de eirculation adoptée hors des rroisements était nettement supérieure. En fait cette dernière vitesse, supérieure à $4 \mathrm{Km} / \mathrm{h}$, est-elle pratiquement réalisable avec les caractéristiques choisies pour les péniches tractées d'un coefficient 0,98 ? $6^{\circ}$ Quant à l'érosion plus forte par automoteurs en des points particuliers (abords des écluses, toumants de la roie navigable, points de reprise de vitesse, etc...) elle est nettement inscrite dans les faits par l'aspect des écluses, par exemple au cours de chômage des canaux. Sans même être spécialistes, et en dehors des ingénieurs, tous les usagers le savent.

Telles sont les observations qu'on peut tirer d'unc première lecture du mémoire; il peut y en avoir d'autres qu'une étude plus poussée pourrait révéler.

En tout cas, l'objet de ma lettre n'a pas pour but de soulever un «incident», mais simplement d'éviter des conclusions d'ordre pratique à la suite d'essais sur modèles réduits qui n'englobent pas la totalité des faits récls.

Veuillez croire, mon cher Président, à mes sentiments les plus cordiaux.

M. Georges Werr ajoute verbalement que la plupart des canaux sont à section trapézoïdale, et non rectangulaire, que leurs berges sont revetues de sable granuleux ou de végétation, donc assez différentes de colles montrées sur les pholographies; il lui semble assez difficile de déterminer exactement le résultat du passage (1'nn automotenr dont l'hélice ne mesure que $1,90 \mathrm{~m}$ de diamètre sur des modèles aussi réduits que coux que M. Lxenumper a expérimentés (similitude de Froude) et it la suite du passage de 30 bateaux, ce qui correspond, par exemple, au trafic du Canal de Saint-Quentin en $1 / 4$ de journée. En conelusion, M. Georges Wen ne met pas en donte les résultats scientifiques des essais relatés par M. LhenmTe, mais il fail toutes réserves sur la transposition de ces résultats sur un plan rivant el mobile, comme celui des canaux et des bateaux.

M. le Président remarque trutefois que les essais de laboratoire domnent en général de bons résultats pratiques, notamment pour les problèmes d'hydraulique maritime; il faut, évidemment dans certains cas, prendro wne echelle assez grande par exemple, $1 / 12$ au lieu de $1 / 2.5$, dans le cas des perrés du Canal de Suez. Il semble, d'ailleurs, très difficile d'étudier un phénomène donmé, comme le croisement de bateaux, la comparaison de l'antomoteur avec les péniches, autrement que sur un modèle. En ce qui concerne le creusement des canaux par le fait du passage des automoteurs, il est curieux de constater que le canal de Bourbourg s'est creusé en triangle alors que le canal de Suez prend la forme en cuiller».

M. Lhennurre résume la lettre ci-après qu'il a adressée le 24 juin à $M$. le Président de Rouvilue, en réponse aux remarques de M. WELL.

\section{Réponse de M. LHERMITTE :}

I. - VAleUR DES CONClustons sur la COMPARAISON DES ÉROSTONS DUES AUX BATEAUX AUTOMOTEURS ET TRACTÉS

\section{Réponse au $1^{\prime \prime}$ : Point de vue des principes et au $1^{\prime \prime}$ : Point de vue des faits.}

Les essais effeetivement effectués dans le but d'étudier l'efficacité de certains dispositifs de revêtement pour lutter contre l'érosion des berges, ont également été ef́fectués à titre de comparaison sur des sections nou revêtues, ainsi qu'il est bien préeisé dans la communication.

Les observations que nous avons faites a ce propos ont permis d'établir certains faits peu connus que nous avons cru bon de verser au dossicr du problème de l'érosion des voies navigables. Ils ont, en particulier, fait apparaître le fait fondamental et, je crois, jamais mis cn lumière de façon évidente, à savoir que les péniches 
automotrices ont, au cours de déplacements à vitesses égales, une action d'érosion sur les berges plus faible que les péniches tractées. Je crois que ce phénomène est établi sur des bases suffisantes à la suite des expériences que nous avons effectuées.

\section{II. - Caractéristiques des essais.}

Réponse aux $2^{\circ}$ et $3^{\prime \prime}$ : Point de vue des principes et $a u x 2^{\circ}$ et $3^{\circ}$ : Point de vue des faits.

Les essais ont effectivement été effectués dans des cas particuliers destinés à mettre en lumière de façon plus: visible les phénomènes d'érosion. C'est pourquoi nous avons utilisé des péniches de bloc coefficient élevé.

Il importe toutefois de remarquer que si nous avons pris le maximum du bloc coefficient pour les péniches tractées, nous avons également pris, pour les automoteurs, un maximum qui n'est actuellement pas atteint.

En ce qui concerne les profondeurs, je ne pense pas que les différences de protondeurs changent beaucoup les résultats, mais ce serait, de toute façon, des essais intéressants à entreprendre afin de compléter ceux eiffectués.

Enfin, je ne vois pas queile conclusion erronée pourrait apporter à nos résultats le fait que les croisements nnt toujours été provoqués au même endroit. Ainsi que nous l'avons toujours précisé, cette méthode est la seule permettant de mettre en lumière les phénomènes propres au croisement des péniches et ceux-ei sont de toute facon, des phénomènes secondaires.

\section{TII. - Erosion due AUX AUtomoteURs.}

\section{Réponse au $4^{\prime \prime}$ : Point de vue des principes, et $\alpha u 6^{\circ}$ : Point de vue des faits.}

Nous avons insisté largement, dans les trois demiers paragraphes de $\mathrm{C}-3$, de notre rapport, sur les dangers particaliers d'érosion due, d'une part, aux sillages de l'hélice et, d'autre part, anx automoteurs lèges qui ne respectent pas la limitation de vitesse.

Nous rappelons que le but de l'étude était essentiellement d'étudier les érosions de batillage et que nous n'avons pas inclus dans le cadre de cette étude les prom blèmes d'érosion spéciaux aux automoteurs.

II est bien connu, comme l'indique M. WLIL, que les érosions des automoteurs, lors du démarrage des peniches, abords des écluses, sont extrêmement intenses. Ceei rejoint d'ailleurs l'idée de zones, exceptionnellement clevées, de turbulence, que nous avons développèe dans notre communication.

\section{IV. - Approfondissement jes Canaux.}

Je n'ai jamais pensé que le creusement d'un canal soil au moins aussi intéressant que son élargissement. I'ai simplement indiqué (D-3) qu'un approfondissement central de la section du canal est très souvent rentable et j'ai précisé que cet approfondissement était particulièrement justifié dans le cas, où par suite dés conditions naturelles, il était impossible d'élargir la largeur en surface du canal.

\section{V. - VITESSE de CIRGULATION DES PÉNiches aU COURS DES ESSAIS.}

II a été indiqué au cours de la communication, et je m'excuse que ceci n'ait pas été reproduit dans le texte polycopié, que les essais ont été effectués pour des viiesses de traction des péniches de $6 \mathrm{~km} / \mathrm{h}$ et de $5,5 \mathrm{~km} / \mathrm{h}$.

Nous avons effectué, d'autre part, des essais de résistance à l'avancement des péniches et nous pouvons préciser que, pour les formes de canaux utilisés, la vitessclimite de péniches tractées d'un coefficient de 0,98 s'établit aux environs de $7 \mathrm{~km} / \mathrm{h}$ a $7.5 \mathrm{~km} / \mathrm{h}$, pour le type de canaux expérimentés.

De plus, M. Lnermite précise verbalement que, d'après l'échelle des temps, 30 passages en maquette correspondent à beaucoup plus en nature; que, d'autre part, ce taux a été choisi parce qu'au cours du tarage du modèle, la comparaison entre les déformations sur modèles et dans la nature a montré qu'il correspondait à un quasi profil d'équilibre; enfin que les essais faits dans le cas des berges revêtues correspondent à un canal que luí a fait visiter M. VADOT au nord de Nancy, dont une partie des berges est en terrain graveleux, ce qui correspond à l'étude théorique, c'est-à-dire à des canaux entièrement en matériaux pulvérulents déposés.

\section{Intervention de M. SUQUET :}

(SO.GR.E.A.H.)

$1^{n}$ M. Sveuet demande a M. LHermitte comment il a mis en évidence les couches limites existant sous le fond de la peniehe et sur le fond du canal et indique les constatations de la SO.GR.E.A.H. à ce sujet :

Un micromoulinet place entre le tond du navire el to fond du canal indique d'abord une vitesse en sens inverse de la direction de marehe du navire, puis sous certaines conditions le sens de cette vitesse s'inverse en sorte qu'un bouchon d'eau semble accompagner le navire. Cela pourrait être attribué soit à un phénomène de couche limite, soit à un décollement à l'arrière du navire.

$2^{\circ}$ A propos de la différence de contre-courant constalé entre un automoteur at un navire remorqué, $M$. Suquer indique qu'un mieromoulinet place au voisinage de la muraille da navire et à une profondeur égale sensiblement à la moitié du tirant d'eau, a montré que les contre-courants étaient plus grands pour le navire autopropulsé que pour le navire remorqué; la difference ponvant atteindre $50 \%$ dans les cas extrêmes (seetion faible et vitesse voisine de la « vitesse limite 》 de Schijf). La mesure du courant en surface, faite seulement dans le cas du navire autopropulsé et pour des sections fair bles, a donné des valeurs très sensiblement égales à différentes immersions, et ceci dans de très nombreux cas.

$3^{\circ}$ M. Suquet est d'accord avec M. Lhermtre sur l'importance du defferlement sur les berges; l'onde deferlante appelée "volute» sur le Canal de Suez a un effet primordial sur l'érosion de la «banquette on «risberme $»$, ceei d'autant plus que la profondeur de celle-ci est plus fable.

Il est également d'accord sur l'intérêt de l'approfondissement d'un canal. De façon plus précise, ì section constante, un approfondissement est plus «payant» qu'un élargissement au point de vue de la diminution des phénomènes hydrauliques et de l'érosion, dans le cas de valeurs du rapport de la section du canal au maitiecouple du navire voisin de 5 et pour un pied de pilote au repos ayant de $1 / 4$ à $1 / 5$ de la profondeur.

$4^{\circ}$ A propos de l'étude de l'équilibre des perrés dans le canal de Suez, M. SuQuer indique que la SO.GR.E.A.H. avait bien précisé à la Compagnie, des te début des essais, que J'étude complète des perrés necessiterait une schematisation it une echelle beacoup plus grande.

M. Ie Président rappelle gue les derniers résultats obtenus par la SOGR.E.A.H. pour la protection des perres du Canal de Suez ont conduit Ia Compagnie à abaisser, énormément les banquettes $(2 \mathrm{~m}$ à $2,50 \mathrm{~m}$ audessous du plan d'cau) et à les faire très étroites $(5 \mathrm{~m}$ au plus).

A propos de l'observation de M. Suquet sur la différence d'effet d'un navire tracté et d'un navire automoteur, M. le Président indique que ceci avait conduit $M$. I'Inspecteur général Outnex à suggérer non que les navires ne passent plus avec leurs machines, mais à les faire ajder par des remorqueurs.

\section{LHERMITTE répond à M. SUQUET :}

$1^{\circ}$ Les couches limites sur le fond ont dé nises an ividence par les observations relatives aux essais d'ero. 
sion ciddessus relatés, mais elles ont èté surtout mises en évidence au cours d'autres essais relatifs à la determination de la rèsistance à l'avancement : la discontinuité qui se présente pour les courbes de résistance relatives à plusieurs enfoncements $(1,80 \mathrm{~m}$ à $2,20 \mathrm{~m})$ était d'autant plus sensible que l'échelle était plus petite; les méthodes colorimetriques ont conduit a rendre responsable de ce fait la génération des couches-limites qui arrivent à se rejoindre et cela a permis d'expliquer que la résistance à l'avancement du modèle est, toute chose égale par ailleurs, d'autant plus importante que l'échelle est petite. Les résultats communiqués par M. Suover sont interessants en ce qu'ils recoupent cette interprotation.

$2^{\circ}$ Les courants de retour que M. Stouer signale comme étant les plus importants à mi-hauteur ont toujours été mesurés en surface en vue de l'étude de l'érosion des berges: les essais ont été faits d'une part sur des péniches à traction, (caisses à savon), dont le bloc coefficient est de 0,98 , qui ont, à l'arrière un sillage d'eau mort, et une alimentation en eau difficile, d'autre part sur des navires de forme plus affine dont lalimentation de l'hélice est facilitée. Il semble que, aussi bien sur les canaux de navigation maritime que sur les canaux de navigation fluviale, l'hélice a tendance à créer dans l'aspiration une certaine composante verticale qui est particulièrement importante pour les peniches qui circulent sur les canaux de navigation fluviale.

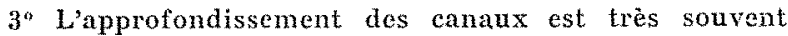
rentable; celui qui a été obtenu à Suez par le seul fait de la navigation a semblé être un gain; la déformation du Canal de Bourbourg, signalée par M. Wes, venait peut-être du fait que la pente des berges avait perda son equilibre et provoqué un certain comblement latéral. Mais si lon admet des protections partielles des berges, l'approfondissement, après enlèvement d'une partic des matériaux, augmente la rentabilité du canal et diminue les risques d'érosion. L'étude de la section économique qui donne le moins d'entretien et qui est la plus favorable à la navigation mériterait d'être aite de facon systématique et, à ce point de vue, le modèle réduit pourrait ctre un apport puissant.

En réponse a une nouvelle question de $M$. Suquer, N. Lhenmite précise que le rapport maitre-couple canal est de l'ordre de $1 / 5$ et que les essais des péniches flamandes étudiées ont été faits à des profondeurs de $1,80 \mathrm{~m}_{*}$ $2 \mathrm{~m}$ et $2,20 \mathrm{~m}$, correspondant, suivant les données de $\mathrm{M}$. VADOT, au plan d'aménagement actucllement en cours de réalisation dans l'Est de la France.

Intervention de M. BINDEL (Bassin des Carènes) :

M. Binnet, remarquant que le régime permanent d'écoulement autour de la carène est très long à s'établir en canal, et que ceci nécessite de disposer pour les essais d'un canal relativement long, demande à M. Lhernitre: de lui indiquer quel etait le rapport:

\section{$\frac{\text { longueur du camal }}{\text { longueur du modèle }}$}

au cours des essais.

D'autre part, dans les essais d'automoteurs, il est nécessaire de faire travailler les hélices dans les mêmes conditions que sur le réel; M. Bindel demande done à M. LhERMTTE de lui indiquer si, au cours des essais, on a tenu compte du fait que le modèle est relativement plus résistant que le réel et si l'on a appliqué aux automoteurs une scorrection de frottement $»$, force de trattion qui s'ajoute a la poussée des hélices pour réaliser l'autopropulsion du modèle.

\section{LHERMITTE répond à M. BINDEL :}

$1^{\circ}$ Le canal d'expérience avait une longueur de $57 \mathrm{~m}$ pour une péniche d'environ $3 \mathrm{~m}$ de longueur; de plus, linstallation comportait d'abord une section de mise en vifesse, dont le rapport au mâtre-couple etait beaucoup plus grand (plus du double) que dans la réalité, ce aui permettait d'obtenir rapidement la vitesse limite pour passer dans la section «en dur $\gg$ qui représentait exactement le canal; ainsi pouvait-on homogénéiser la vitesse dans la section d'étude aui avait 30 metres de Iongueur, dont 20 mètres réalisés en fonds aftouillables.

$2^{\circ}$ En ce qui concerne la correction de frottement dans le cas des automoteurs, celle-ci était réalisée par un effort de traction constant et pour lequel on a verifié, au cours des essais, que le point de fonctionnement cor. respondait à un point de Ia courbe d'autopropulsion.

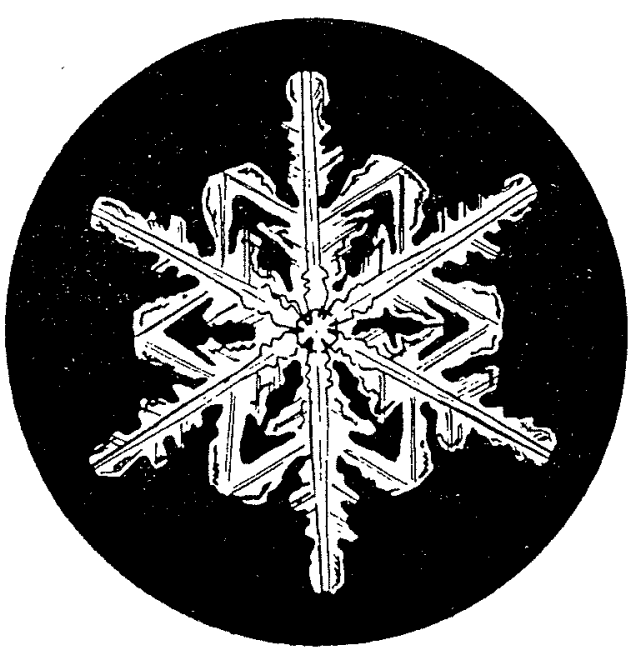

